\title{
A ZERO-ONE, BOREL PROBABILITY WHICH ADMITS OF NO COUNTABLY ADDITIVE EXTENSIONS
}

\author{
LESTER E. DUBINS ${ }^{1}$
}

\begin{abstract}
There is a subsigma-field of the Borel subsets $B$ of the unit interval which supports a countably additive, two-valued, probability which cannot be extended to $B$ so as to remain countably additive.
\end{abstract}

This note notes the existence of a countably additive probability $P$ defined for a sigma-field of Borel subsets of $\mathbf{R}^{\infty}$ which satisfies this peculiar property: If $\omega=$ $\left(\omega_{1}, \omega_{2}, \ldots\right) \in \mathbf{R}^{\infty}$ is $P$-distributed, then, for every real number $t$, with $P$-probability $1, \omega_{n}=t$ for some $n$.

Formally, let $E_{t}$ be the set of $\omega \in \mathbf{R}^{\infty}$ such that $\omega_{n}=t$ for at least one positive integer $n$, and let $U$ be the sigma-field generated by the family $\left\{E_{t}, t \in \mathbf{R}\right\}$.

PROPOSITION 1. There is one, and only one, countably additive probability $P$ defined on $\mathcal{U}$ such that $P\left(E_{t}\right)=1$ for all $t$.

Proof. As is easily verified, there is one, and only one, finitely additive probability $P_{1}$, defined on the field $₹$ generated by the $E_{t}$ such that $P_{1}\left(E_{t}\right)=1$ for all $t$. Let $F_{i} \in \mathcal{F}, F_{i+1} \subset F_{i}, 1 \leq i<\infty$ with $\inf P_{1}\left(F_{i}\right)=\epsilon>0$. To see that $P_{1}$ is countably additive, it is only necessary to see that $\bigcap F_{i}$ is nonempty. To this end, note first that $\epsilon=1$, so $P\left(F_{i}\right)=1$ for all $i$. Now call a sequence $G_{1}, G_{2}, \ldots$ a selection if, for each $n, G_{n}$ is $E_{t(n)}$, where $t(1), t(2), \ldots$ is a sequence of indices, and then verify: (a) if $G_{1}, G_{2}, \ldots$ is any selection, then $\bigcap G_{n}$ is nonempty; and (b) there is a selection $G_{1}, G_{2}, \ldots$ such that $\bigcap G_{n} \subset \bigcap F_{i}$.

For each real number $t$ and positive integer $n$, let $E_{t, n}$ be the set of all infinite sequences whose $n$th coordinate has the value $t$, and let $\mathcal{V}$ be the sigma-field generated by the collection of all $E_{t, n}$. Since, for each $t, E_{t}$ is the union of the $E_{t, n}, E_{t} \in \mathcal{V}$, so $U \subset \mathcal{V}$.

A probability $Q$ on a sigma-field is purely finitely additive if there is a denumerable collection of elements of the sigma-field, each of $Q$-probability zero, whose union has $Q$-probability one.

LEMMA 1. Let $Q$ be a probability defined on $\mathcal{V}$, or on any $\mathcal{W}$ which includes $\mathcal{V}$, and suppose that $Q\left(E_{t}\right)=1$ for a nondenumerable set of $t$. Then $Q$ is purely finitely additive.

PROOF. For fixed $n$, the set of $E_{t, n}$ are disjoint and, therefore, the set, $T_{n}$, of $t$ such that $E_{t, n}$ has positive $Q$-probability is countable. Consequently, the union, $T$, of the $T_{n}$ is also countable. So there certainly is a $t$ not in $T$. For such a $t$, $E_{t, n}$ has $Q$-probability zero for all $n$, but their set-theoretic union, namely $E_{t}$, has $Q$-probability 1 .

Received by the editors December 21, 1981 and, in revised form, January 20, 1982.

1980 Mathematics Subject Classification. Primary 28A05, 28A35, 60A35.

${ }^{1}$ Research supported by National Science Foundation Grant MCS-80-02535. 
THEOREM 1. Let I be any complete, separable, metrizable space with a continuum of points. Then there is a $(U, P)$ where : (a) $U$ is a subsigma-field of the Borel subsets $B$ of $I$; (b) $P$ is a countably additive, two-valued probability defined on $U$; (c) every probability on $B$ which agrees with $P$ on $U$ is purely finitely additive.

ProOF OF TheOREM 1. For $I=\mathbf{R}^{\infty}$, Proposition 1 , together with Lemma 1 , implies the conclusion. The conclusion for any $I$ follows for, as is well known, $(I, B(I))$ is isomorphic to $\left(\mathbf{R}^{\infty}, B\left(\mathbf{R}^{\infty}\right)\right)$.

ACKNOWLEDGEMENTS. I thank Dev Basu who first told me about a countably additive $P^{\prime}$ on $U$ under which the $E_{t}$ are mutually independent and nontrivial which, therefore, cannot be extended to be countably additive on $B\left(\mathbf{R}^{\infty}\right)$ since $L_{2}$ of such a $P^{\prime}$ is nonseparable. I also thank Benjamin Weiss who recently revived my interest in this phenomenon.

UNIVERSITy OF CALIForNiA, Berkeley, CALIForNiA 94720 